\title{
Lipoma Arborescens of the Elbow: Case Report
}

\author{
Dilek BADAY, ${ }^{1}$ Meltem ARAS, ${ }^{1}$ Aysel ÇOLAK, ${ }^{2}$ Şükrü Cem HATIPOĞLU, ${ }^{3}$ \\ Özdamar Fuad ÖKEN, ${ }^{4}$ Sumru ÖZEL ${ }^{1}$ \\ ${ }^{1}$ Depatment of Physical Medicine and Rehabilitation, Ankara Physical Medicine and \\ Rehabilitation Training and Research Hospital, Ankara, Turkey \\ ${ }^{2}$ Department of Pathology, Ankara Numune Training and Research Hospital, Ankara, Turkey \\ ${ }^{3}$ Department of Radiology, Ankara Physical Medicine and Rehabilitation Training and Research Hospital, Ankara, Turkey \\ ${ }^{4}$ Department of Orthopaedics and Traumatology, Ankara Numune Training and Research Hospital, Ankara, Turkey
}

\begin{abstract}
Lipoma arborescens is a rare, benign articular lesion characterized by villous lipomatous proliferation of the synovium caused by replacement of mature fat cells with subsynovial tissue. Although the knee is the most affected joint, lipoma arborescens has also been observed in other locations such as glenohumeral joint, subdeltoid/bicipital bursae, hip, elbow, wrist, and rarely elbow joints. In this article, we report a rare case of lipoma arborescens at the elbow joint in a 30-year-old female patient with cerebral palsy.

Keywords: Arthritis, elbow; lipoma arborescens; magnetic resonance imaging; synovectomy.
\end{abstract}

Lipoma arborescens (LA) is a rare, benign articular lesion caused by diffuse replacement of mature fat cells with subsynovial tissue. ${ }^{1}$ The term "arborescens" means "tree-like appearance" describing the villous morphology. ${ }^{2}$ It has been considered to be a reactive process of the synovial tissue; however, the etiology is still unclear. $^{3}$ It usually presents as a painless and slowly progressive swelling in joint which is commonly monoarticular; however, bilateral and polyarticular involvement were also reported. ${ }^{1-5}$ Laboratory test results are usually normal, but mildly elevated erythrocyte sedimentation rate (ESR) and slightly increased C-reactive protein (CRP) levels may be seen. ${ }^{5}$ Magnetic resonance imaging (MRI) and synovial biopsy have importance for precise diagnosis. ${ }^{4}$ In this article, we present an unexpected and extremely rare intra-articular lesion of the elbow joint which might be of interest for clinicians.

\section{CASE REPORT}

A 30-year-old female patient with cerebral palsy presented to our clinic with complaints of pain on left elbow, bilateral wrist, ankle, and knee joints. She had also suffered from left elbow swelling for about three years. Treatment with nonsteroidal antiinflammatory drugs was not efficient. Patient had no history of trauma, diabetes mellitus or any inflammatory joint diseases as well as no family history of rheumatological diseases. She mentioned that she underwent right knee operation due to pigmented villonodular synovitis three years ago.

Physical examination revealed soft swelling of the left elbow joint. Skin color was normal, local temperature was minimally elevated and left elbow motion was restricted and painful. Both wrists, knees and ankles were mildly swollen and 
tender on palpation. The plain radiography of the left elbow and the wrists did not show any fracture, dislocation, mass lesion or erosions, but mild osteopenia was present. There were degenerative changes in knee joints. Laboratory tests showed that white blood cell level was $5,200 / \mathrm{mm}^{3}$, ESR was $49 \mathrm{~mm} / \mathrm{h}(0-30)$ and CRP level was $4.03 \mathrm{mg} / \mathrm{dL}(0-0.8)$. Anti-cyclic citrullinated peptide antibody, rheumatoid factor, antinuclear antibodies, and anti-double-stranded DNA were all negative. The patient was first diagnosed as seronegative rheumatoid arthritis (RA) but did not fulfill the 2010 American College of Rheumatology and European League Against Rheumatism classification criteria. ${ }^{6}$

Magnetic resonance imaging of the left elbow (Figure 1) was performed and $\mathrm{T}_{1}$-weighted MRI showed high-signal intensity, while the short tau inversion recovery images showed isointensity with fat nodular signal changes on the anterior of proximal radius and distal humerus, respectively. MRI of the right wrist demonstrated synovial hypertrophy and effusion in the radioulnar and intercarpal joints. For exact diagnosis and treatment, open synovectomy of the left elbow was performed. Histopathological examination of the specimen (Figure 2) showed infiltration

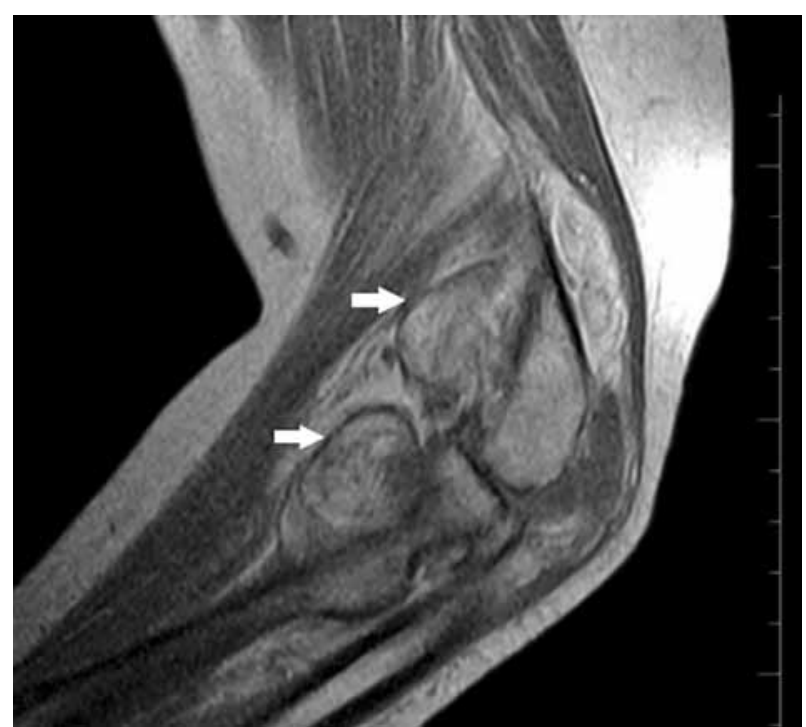

Figure 1. Thirty-year-old patient with pain and swelling of left elbow. Magnetic resonance imaging (sagittal

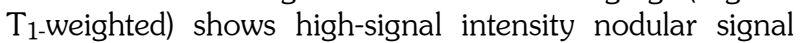
changes on anterior proximal radius and anterior distal humerus (arrows). Synovial hypertrophy and minimal degenerative changes with narrowing of joint space are also shown. of the subsynovial space with mature adipocytes (hematoxylin and eosin stain $\mathrm{x} 40$ ) and the diagnosis was reported as LA.

\section{DISCUSSION}

Lipoma arborescens is a rare, intraarticular lipoma-like lesion characterized by fatty infiltration of the subsynovial tissue., Although the pathogenesis of LA is still unknown; degenerative joint disease, trauma, diabetes mellitus, inflammatory arthritis such as RA, psoriatic arthritis and juvenile spondyloarthropathy have been reported as associated conditions. . $^{1,4}$ Some researchers have proposed the hypothesis that the reason of the transformation of synovial tissue may be the chronic inflammation of the synovium. ${ }^{2}$ The knee is the most affected joint. ${ }^{3,4,7}$ It might present bilaterally but monoarticular involvement is more common. ${ }^{3,5}$ Polyarticular LA with inflammatory synovitis was reported by Santiago et al. ${ }^{8}$ They presented a 29-year-old female patient who had RA treatment due to history of intermittent arthritis of the wrists, knees, ankles and did not benefit from the treatment. ${ }^{8}$ Patient's eventual histopathological diagnosis was LA. ${ }^{8}$ Our patient had swelling on left elbow and pain at the left elbow, both wrists, ankles and knee joints. Physical examination findings and radiological evaluation did not support $\mathrm{RA}$. We were unable to perform synovectomy of the other joints because the patient did

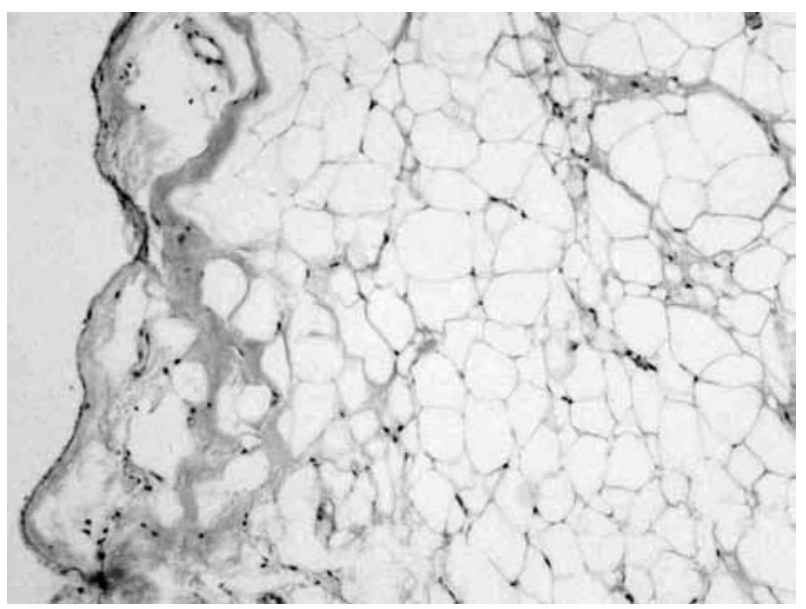

Figure 2. Subsynovial space is infiltrated with mature adipocytes (H-E x 40). 
not consent. We advised follow-up since her clinical, laboratory and radiological findings corresponded to polyarticular LA.

Lipoma arborescens is seen between the ages of nine and 66 with male predominance. ${ }^{3}$ The characteristic clinical feature is painless and slowly progressive swelling joint. Joint pain and motion restriction might develop due to intermittent effusion. ${ }^{1,2,5}$ In our patient, the elbow was painful, swollen, and tender; in addition, joint motion was restricted. Although laboratory tests are usually normal in patients with LA, ESR and CRP levels might be elevated mildly. ${ }^{1,2,5}$ Similarly, ESR and CRP were elevated in our patient.

A soft tissue density might be seen on plain X-ray which is not diagnostic. ${ }^{2}$ Although the characteristic image of MRI, particularly using fat-suppressed or short tau inversion recovery sequences, is diagnostic for LA; ultrasonography, computed tomography and arthrography have been used in diagnosis.,7,9 MRI findings include a synovial mass, fat signal intensity on all sequences, suppression of signal with fat-selective presaturation, joint effusion, and absence of MRI supporting hemosiderin., ${ }^{1,9}$ As the synovial tissue may not be completely infiltrated by mature fat cells, atypical MRI findings may be seen. ${ }^{4}$

Histopathological appearance is the papillary villi infiltrated with mature fat cells. ${ }^{1}$ Mononuclear chronic inflammatory cells may be seen in the overlying synovial membrane and the synovial cells may be reactive with eosinophilic cytoplasm. ${ }^{1}$ In our patient, subsynovial space was infiltrated with mature adipocytes.

Other diffuse proliferative pathologies of the synovium like pigmented villonodular synovitis, synovial chondromatosis, synovial hemangioma, and RA must be considered in the differential diagnosis of LA. ${ }^{3,7}$ To our knowledge, any relationship between pigmented villonodular synovitis and LA has not been reported, although both are proliferative pathologies of the synovium. ${ }^{3}$ Recommended treatment of LA is arthroscopic or open synovectomy. ${ }^{2,7}$ Yttrium-90 radiosynovectomy was reported as another option for the treatment of LA at the knee. ${ }^{10}$
In conclusion, despite the relative rarity of LA, awareness of its clinical and imaging findings is essential to avoid misinterpretation. LA should be considered in patients with swollen and painful joints who have elevated ESR and CRP level.

\section{Declaration of conflicting interests}

The authors declared no conflicts of interest with respect to the authorship and/or publication of this article.

\section{Funding}

The authors received no financial support for the research and/or authorship of this article.

\section{REFERENCES}

1. Kloen P, Keel SB, Chandler HP, Geiger RH, Zarins $\mathrm{B}$, Rosenberg AE. Lipoma arborescens of the knee. $\mathrm{J}$ Bone Joint Surg [Br] 1998;80:298-301.

2. Kamaci S, Doral MN, Ergen FB, Yucekul A, Cil A. Lipoma arborescens of the knee. Knee Surg Sports Traumatol Arthrosc 2014 Apr 22. [Epub ahead of print]

3. Garner HW, Bestic JM. Benign synovial tumors and proliferative processes. Semin Musculoskelet Radiol 2013;17:177-8.

4. Xue J, Alario AJ, Nelson SD, Wu H. Progressive bilateral lipoma arborescens of the knee complicated by juvenile spondyloarthropathy: a case report and review of the literature. Semin Arthritis Rheum 2013;43:259-63.

5. Demir SÖ, Kibar S, Aras M, Köseoğlu F. Lipoma arborescens of the knee: Case report. Rheumatism 2007;22:147-50.

6. Aletaha D, Neogi T, Silman AJ, Funovits J, Felson DT, Bingham CO, et al. 2010 rheumatoid arthritis classification criteria: an American College of Rheumatology/European League Against Rheumatism collaborative initiative. Ann Rheum Dis 2010;69:1580-8.

7. Ranganath $\mathrm{K}$, Rao GB, Namitha. Lipoma arborescens of the elbow. Indian J Radiol Imaging 2010;20:50-2.

8. Santiago M, Passos AS, Medeiros AF, Sá D, Correia Silva TM, Fernandes JL. Polyarticular lipoma arborescens with inflammatory synovitis. J Clin Rheumatol 2009;15:306-8.

9. Feller JF, Rishi M, Hughes EC. Lipoma arborescens of the knee: MR demonstration. AJR Am J Roentgenol 1994;163:162-4.

10. Erselcan T, Bulut O, Bulut S, Dogan D, Turgut B, Ozdemir $S$, et al. Lipoma arborescens; successfully treated by yttrium-90 radiosynovectomy. Ann Nucl Med 2003;17:593-6. 